EPJ Web of Conferences 41, 08020 (2013)

DOI: $10.1051 /$ epjconf/20134108020

(C) Owned by the authors, published by EDP Sciences, 2013

\title{
A New Mechanism for Photosynthetic Energy Transfer
}

\author{
V. Tiwari ${ }^{1}$, W. K. Peters ${ }^{1}$, and D. M. Jonas ${ }^{1}$ \\ ${ }^{1}$ Department of Chemistry and Biochemistry, University of Colorado, Boulder, Colorado 80309- \\ 0215, USA
}

\begin{abstract}
Calculations reveal a new kind of non-adiabatic funnel that electronically enhances anti-correlated vibrational wavepackets on the ground state. These wavepackets replicate all observed $2 \mathrm{D}$ signatures of photosynthetic energy transfer, including one not previously explained.
\end{abstract}

\section{Introduction}

Electronic energy transfer in photosynthetic light harvesting antennas is extraordinarily efficient. Femtosecond 2D spectra have revealed the energy transfer pathways and found quantum coherence between photosynthetic pigments that persists throughout the energy transfer process ( $\sim \mathrm{ps}$ timescales). However, the energy transfer mechanism and the origin of this coherence have remained elusive. Several 2D signatures have been attributed to electronic coherence (EC) and oscillatory electronic state populations (quantum transport, QT) [1-2]. In contrast, others have argued that a $\sim$ ps oscillation lifetime implicates excited state vibrational-electronic wavepackets [3].

We present the first exact non-adiabatic calculations of 2D spectra for a model antenna. In nonadiabatic theory, electronic motion need not instantly follow vibrational motion. For an antenna with two identical pigments, we find that when the pigment vibrational frequency is approximately resonant with the energy gap between excitons (electronic states delocalized over the two pigments), there is a non-adiabatic electronic enhancement of femtosecond impulsive excitation for a special kind of ground state vibrational wavepacket, in which the intramolecular vibration on the donor vibrates out of phase with the intramolecular vibration on the acceptor. These non-adiabatically driven ground state vibrations not only reproduce all observed signatures of EC and QT, but also explain an observed asymmetry between opposite cross-peak oscillation amplitudes not previously explained by theory. On the excited electronic state, anti-correlated vibrations drive rapid electronic energy transfer through a new kind of nested intermolecular funnel, which does not have the wellknown conical topology.

\section{Model and Calculation}

We use a standard Hamiltonian with identical harmonic intramolecular vibrations, $q_{A}$ and $q_{\boldsymbol{B}}$, on pigments $A$ and $B$. For both pigments, the equilibrium vibrational coordinate increases by $d$ upon electronic excitation. The antenna protein shifts pigment electronic energies to $E_{A}$ and $E_{B}$. The two states with one pigment excited have a coordinate independent coupling $J$. The delocalized, anticorrelated vibration of the pigment pair, $\left(q_{A}-q_{B}\right) / 2^{1 / 2}$, which tunes the electronic energy difference between pigments, is included when solving for the non-adiabatic vibrational-electronic states. This unrestricted use, distribution, and reproduction in any medium, provided the original work is properly cited. 
must be repeated for an ensemble with an inhomogeneous distribution of energy gaps, $E_{A}-E_{B}$. Correlated vibrations of the pigment pair, $\left(q_{A}+q_{B}\right) / 2^{1 / 2}$, are not coupled to the non-adiabatic dynamics and can be treated analytically. The nonadiabatic $3 \mathrm{D}$ nonlinear impulse response is calculated by a sum over states formula, and multiplied by the analytical $3 \mathrm{D}$ response for correlated vibrations to get the total 3D response. 2D spectra are calculated by a 3DFT algorithm.

\section{Results}

Fig. 1 shows the electronic potential energy surfaces and levels for the isolated pigments and the Born-Oppenheimer adiabatic states (which are partially delocalized excitons) as a function of the anti-correlated vibrational coordinate. The color indicates electronic state character, which of course does not vary for the isolated pigments, and varies slowly for the adiabatic states. In contrast, the exact non-adiabatic states (levels not attached to curves) have a mixed electronic character that varies rapidly with coordinate.
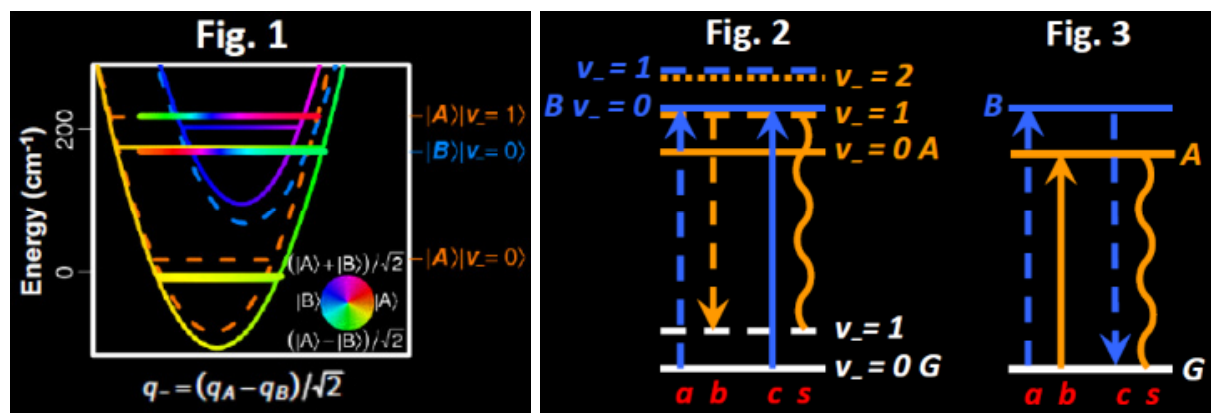

Fig.1. Pigment (dashed), adiabatic (thin solid) and non-adiabatic (thick solid) levels and electronic character along the anticorrelated vibrational coordinate. The wheel shows how color maps onto electronic wavefunction character.

Fig. 2 and Fig. 3. Feynman pathways for cross-peaks in the $2 \mathrm{D}$ spectrum arising from nonadiabatic excitation of ground state vibrations (Fig. 2) and pure electronic coherence (Fig. 3). These two cross-peaks have very similar signatures because the frequencies (arrow length) and transition dipole directions (orange vs. blue arrow color) are all the same.

In Fig. 1, $v_{-}$is the vibrational quantum number for the anti-correlated vibration. The nonadiabatic mixing of the $\mathrm{v}_{-}=1$ state on $A$ with the $\mathrm{v}_{-}=0$ state on $B$ shown in Fig. 1 means that this mixed state has strong transitions with $\Delta \mathrm{v}_{-}=0$ character to both $\mathrm{v}_{-}=0$ and $\mathrm{v}_{-}=1$ on the ground electronic state. The $2 \mathrm{D}$ spectra for this model have four main peaks: diagonal peaks DP1 at $\left(\left|\omega_{A}\right|,\left|\omega_{A}\right|\right)$ and DP2 at $\left(\left|\omega_{B}\right|,\left|\omega_{B}\right|\right)$, plus cross peaks CP12 at $\left(\left|\omega_{B}\right|,\left|\omega_{A}\right|\right)$ and CP21 at $\left(\left|\omega_{A}\right|,\left|\omega_{B}\right|\right)$. Fig. 2 shows how anti-correlated ground state wavepackets are excited in a $2 \mathrm{D}$ experiment and give rise to strong oscillations of CP12 in a rephasing (R) 2D spectrum (photon echo pulse sequence). The first and last frequencies on the diagram give the excitation and detection frequencies in the 2D spectrum. Dipole directions for the 4 transitions are indicated by color (using the color wheel in Fig. 1) and are identical to those involved in excitation of electronic coherence on the excited state, shown in Fig. 3. As a result, these ground state vibrational wavepackets naturally reproduce signatures previously attributed to EC and QT. Figure 4 shows three signatures in rephasing $2 \mathrm{D}$ spectra: the $180^{\circ}$ phase relation between CP12 and CP21 (EC); the oscillatory DP in R 2D spectra (QT); and the $90^{\circ}$ relative phase between DP2 and CP12 (QT) are reproduced by anti-correlated ground state vibrations. All other observed EC/QT signatures are also reproduced by these non-adiabatically excited ground state vibrations [4].

In the non-adiabatic model, the above specific phase relationships only arise when a pigment vibration with displacement $d \neq 0$ has a frequency near resonance with the excitonic energy gap of the dimer. The required resonance has been checked by comparing vibrational frequencies in the 
fluorescence spectrum of $B C h l a$ (the FMO antenna pigment) to cross-peak oscillation frequencies in the 2D spectrum of FMO. 7 out of 9 match.
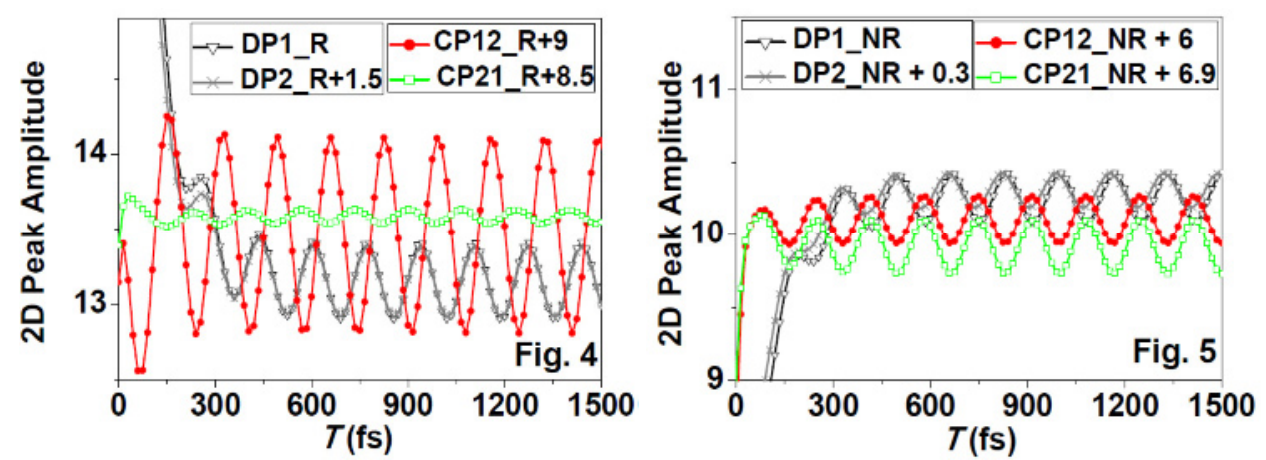

Figs. 4-5. 2D Peak amplitude oscillations in the ground state Rephasing (R, Fig. 4) and NonRephasing (NR, Fig. 5) 2DFT spectra, respectively. The calculations use the small FC displacements of $B C h l a$ monomers in FMO and a typical choice of $\Delta=150 \mathrm{~cm}^{-1}$, and $J=66$ $\mathrm{cm}^{-1}$ for FMO, such that the excitonic energy gap is resonant with the vibrational frequency of $200 \mathrm{~cm}^{-1}$. The strong non-adiabatic coupling results in long-lived oscillations at the resonant vibrational-electronic frequency.

In contrast to EC and QT models, which predict equal amplitude oscillations for CP12 and CP21 in a rephasing 2D spectra, ground state Feynman pathways for CP21 in the non-adiabatic model involve unfavorable steps with $\Delta \mathrm{v}_{-}=1$ and without electronic-vibrational resonance. Fig. 4 shows that oscillations are $\sim 14 \mathrm{x}$ weaker for $\mathrm{CP} 21$ than $\mathrm{CP} 12$. This asymmetry has been reported for cryptophyte marine algae antenna PE545 [2]; only oscillations for CP12 have been reported for FMO [1] and the cryptophyte antenna PC645. The new mechanism predicts other differences with EC/QT and adiabatic vibration models that are consistent with available data and open to experimental test, such as the CP12 oscillation amplitude asymmetry between rephasing (R) 2D spectra in Fig. 4 and nonrephasing (NR) 2D spectra in Fig. 5. Interestingly, for the non-adiabatic model, these experimentally observed signatures are all obscured by excited state coherence as long as it persists.

\section{Conclusions}

Our results suggest that antenna proteins arrange pigment energies and couplings for excitonic energy gaps to be in resonance with a particular vibration on a selected pigment. The pigment vibration must be slightly displaced upon electronic excitation. Through specific resonances with slightly displaced vibrations, the non-adiabatic coupling between vibrations and electrons generates a new kind of funnel that lacks the conical topology of molecular conical intersections and funnels, yet changes the electronic wavefunction within half a vibrational period for all accessible vibrational coordinates. Such nested intermolecular funnels are unavoidable, dictated by independently measured parameters, and provide a new mechanism for photosynthetic energy transfer.

\section{References}

1. G. Panitchayangkoon, D.V. Voronine, D. Abramavicius, J.R. Caram, N.H.C. Lewis, S. Mukamel, G.S. Engel, Proc. Natl. Acad. Sci. USA, 108, 20908 (2011)

2. E. Collini, C.Y. Wong, K.E. Wilk, P.M.G. Curmi, P. Brumer, G.D. Scholes, Nature, 463, 644 (2010)

3. N. Christensson, H.F. Kauffmann, T. Pullerits, T. Mančal, J. Phys. Chem. B, 116, 7449 (2012)

4. V. Tiwari, W.K. Peters, D.M. Jonas, Proc. Natl. Acad. Sci. USA, submitted 